\section{La complejidadr una} perspectiva fflosófica
La complejidad: una perspectiva filosófica| Revista

Virtual Universidad Católica del Norte, 64, 281-308

ISSN: 0124-5821 (En línea)

Urrego-Marín, M. L., y Ocampo Osorio, C. (septiembre-diciembre, 2021). La complejidad: una perspectiva filosófica y multidisciplinar en las ciudades inteligentes. Revista Virtual Universidad

Católica del Norte, (64), 281-308. https://www.doi.org/10.35575/rvucn.n64a11

\title{
La complejidad: una perspectiva filosófica y multidisciplinar en las ciudades inteligentes
}

\section{Complexity: a philosophical and multidisciplinary perspective on smart cities}

\section{María Lucelly Urrego-Marín}

$\mathrm{PhD}(\mathrm{c})$ in Complex Thinking

Facultad de Economía y Ciencias Administrativas, Corporación Universitaria Minuto de Dios

Medellín, Colombia

murregomari@uniminuto.edu.co

Orcid: https://orcid.org/0000-0003-4363-1366

CvLac: https://scienti.minciencias.gov.co/cvlac/visualizador/generarCurriculoCv.do?cod_rh=000001520

\section{Cristina Ocampo Osorio}

Magister en Gestión de Organizaciones

Centro de Investigaciones, Institución Universitaria Escolme

Medellín, Colombia

cies4@escolme.edu.co

Orcid: https://orcid.org/0000-0002-2137-6336

CvLac: https://scienti.minciencias.gov.co/cvlac/visualizador/generarCurriculoCv.do?cod_rh=0001765497

Recibido: 17 de enero de 2021

Evaluado: 19 de marzo de 2021

Aprobado: 21 de abril de 2021

Tipo de artículo: Reflexión

\section{Resumen}

La concepción de complejidad es una variable inherente a las ciudades inteligentes, por ello debe establecerse la diferencia entre los diferentes predecesores de la cultura occidental y oriental, y cómo aportan los pensadores que podrían considerarse pioneros de la complejidad a tales teorías, desplegados a través del tiempo. En este sentido, el artículo consideró una revisión literaria en torno a las teorías de diversas culturas y los periodos filosóficos y corrientes que, desde su 


\section{La complejidadr una} perspectiva fflosófica
La complejidad: una perspectiva filosófica| Revista Virtual Universidad Católica del Norte, 64, 281-308

ISSN: 0124-5821 (En línea)

intencionalidad, expresan un tipo de contexto frente a la concepción de las ciudades inteligentes; concepciones de nuevo conocimiento que de alguna forma sobrepasan, en diversos aspectos y formas, la manera de ver la realidad, y están encaminados a identificar el azar, la incertidumbre o el devenir. Para ello, se planteó una metodología que se ejecutó en 5 fases (referenciación, evaluación, propuesta metodológica, formulación y validación), identificando teorías, en la fase de referenciación, tales como la teoría del caos, la teoría algorítmica de la información, la teoría de simplicidad y la teoría general de sistemas; todas ellas contribuyeron a entender las diversas dinámicas presentes y posibles de las ciudades inteligentes, al tiempo que favorecieron la comprensión de las problemáticas sociales y retos que deben enfrentar estos territorios urbanos.

Palabras clave: Ciudades Inteligentes; Pensamiento Complejo; Pensamiento Filosófico; Pioneros de la Complejidad; Teorías de la complejidad.

\begin{abstract}
The conception of complexity is an inherent variable in smart cities, which is why the difference must be established between the different predecessors of Western and Eastern culture, and how thinkers who could be considered pioneers of complexity contribute to such theories, deployed through the weather. In this sense, the article considers a literary review around the theories of various cultures and the philosophical and current periods that, from their intentionality, express a type of context against the conception of smart cities; conceptions of new knowledge that somehow surpass, in various aspects and forms, the way of seeing reality, and are aimed at identifying chance, uncertainty or future. For this, a methodology was proposed that was executed in 5 phases (referencing, evaluation, methodological proposal, formulation and validation), identifying theories, in the referencing phase, such as chaos theory, algorithmic information theory, theory simplicity and general systems theory; All of them contributed to understanding the various present and possible dynamics of smart cities, while favoring the understanding of the social problems and challenges that these urban territories must face.
\end{abstract}

Keywords: Smart Cities; Complex Thought; Philosophical Thought; Complexity Pioneers; Complexity Theories. 


\section{Introducción}

Actualmente las ciudades están identificando estrategias de ecosistemas inteligentes, que se renuevan para tratar de establecer y precisar el mapa de ruta a seguir, para certificar la viabilidad y sostenibilidad de los territorios. Sin embargo, considerar esta variable dentro de esta dinámica, no es una tarea fácil, pues es preciso adherir, a todo esto, el componente tecnológico que se caracterice por el uso eficiente y responsable de los datos; y, por otro lado, el diseño e implementación de políticas públicas capaces de responder a las carencias básicas institucionales y de sus pobladores, con el fin de asegurar el progreso urbano sostenible, a partir del diseño de modelos locales eficaces, que tracen y supervisen de forma distintiva y particularizada el progreso escalonado en cada territorio.

Conviene señalar que autores como Wiener (1948), Bertalanffy (1968), Morin (1999b), Moreno (2003), y Osorio et al. (2008), realizan una revisión de literatura en torno a la concepción de antecesores relevantes de la cultura oriental y la cultura occidental, el desarrollo de los diferentes periodos filosóficos, desde la edad antigua hasta la edad contemporánea, y con ello la contextualización de los pioneros de la complejidad entre 1948 y 2008, su evolución y algunas teorías incorporadas de la complejidad, como la teoría del caos, la teoría algorítmica de la información, la teoría de la simplicidad y la teoría de sistemas, las cuales aportaron de forma significativa al proceso investigativo realizado.

Partiendo de esta premisa, como lo mencionan Wiener (1948), Bertalanffy (1968), Morin (1999b), Moreno (2003), y Osorio et al. (2008), es preciso considerar el análisis de la variable poblacional. Situación que ha generado preocupación en los dirigentes de los grandes centros urbanos, pues viene evolucionando considerablemente y de forma desmesurada, afectando los sectores económicos, la movilidad, el manejo adecuado de los recursos naturales, la educación, la prestación de los servicios de salud, y deteriorando la calidad de vida de los ciudadanos.

Cuando se analizan las nuevas opciones filosóficas, aplicables hoy a las ciudades inteligentes, se parte del hecho de que definir las ciudades inteligentes no es un término nuevo, pues estos centros urbanos siempre han existido, y como tal parten del hecho del dinamismo de 
sus ciudadanos, en un entorno donde se evalúan escenarios que posibilitan el progreso y establecen interacciones entre sus actores y el entorno, para garantizar la competitividad y el uso eficiente y adecuado de sus recursos. Con todo esto, se observa una innegable participación de diferentes actores sociales que hacen parte, indisoluble e infaltable, de un sistema, y que como tal está compuesto por elementos interrelacionados entre sí, como un todo.

Ciertamente, al hacer referencia a estos centros urbanos, se debe, sin lugar a dudas, considerarlos por sus características particulares como sistemas complejos que parten del hecho de que, para ser sostenibles y sustentables, deben conocer primero cómo es la forma de interactuar entre ellos y, consecuentemente, centrarse en su interacción con el entorno, para definir procesos y estrategias que le permitan el control de sus recursos, en aras de alcanzar la sostenibilidad del territorio. En esta medida, el artículo realiza una aproximación a los diferentes periodos filosóficos y a los antecesores de la cultura oriental y occidental, con el fin de establecer una línea de tiempo que permita comprender la evolución de estas regiones y las diferentes variables que las han llevado a convertirse en espacios que generan una calidad de vida digna para sus moradores; a la vez, entender desde las múltiples disyuntivas, de estas corrientes filosóficas, los nuevos enfoques creados e identificables en las actuales dinámicas de ciudad, que si bien no se expresan taxativamente en las teorías citadas, sí están tácitamente sugeridas como alternativas dentro de la inmensa riqueza de la complejidad misma, los territorios y el hombre, presente siempre en ellos.

\section{Metodología}

El diseño investigativo estuvo enmarcado dentro de un enfoque documental, que propuso una metodología cualitativa-inductiva, con el fin de cumplir con los objetivos propuestos. En este sentido, se desarrollaron 5 fases: 1) fase de referenciación: se realizó una búsqueda exhaustiva en bases de datos especializadas; 2) fase de evaluación: se realizaron entrevistas a expertos, con el fin de identificar componentes de sustentabilidad; 3) fase de propuesta metodológica: estuvo encaminada a evaluar la sustentabilidad en la ciudad caso de estudio, tomando como punto de partida la concepción de Smart City; 4) fase de formulación de la metodología; y 5) fase de validación de la propuesta metodológica; en esta fase, se validó con los expertos cómo la definición de Smart City aportaba a los diferentes contextos, llevados a cabo en la ciudad analizada. 
Como resultado de lo señalado, la metodología permitió realizar una aproximación de las diversas contextualizaciones, presentes en las fases propuestas, que, desde lo planteado por los autores referenciados al final del texto, ponen de relieve el grado de importancia que ha ido ganando el término en dicha línea de tiempo. Además, las diferentes adaptaciones que implican el manejo de tecnologías de información y comunicación -TIC-, y las normativas existentes para el manejo de los datos en contextos económico-sociales, en aras de definir los territorios como autosuficientes y sostenibles.

Así mismo, identificar las diferentes variables que hacen parte del componente sistémico urbano, con el fin de generar sinergias entre los diferentes actores que en el intervienen. Esta no es una tarea fácil, pero es ahí donde intervienen las disciplinas de la complejidad, las cuales contribuyen a comprender el funcionamiento escalonado y visualizar las posibles soluciones que ayuden a los territorios a disminuir los altos índices de pobreza.

Tal y como plantean Rauch (1993) Caragliu et al. (2011) y Zárate (2010), es notoria la preocupación que genera en las comunidades el hecho de reducir el índice de pobreza en sus territorios, y que genere actividades que otorguen valor a sus empresas y ciudadanos para diversificar y emplear, de forma eficiente, sus recursos, convertirlos en territorios competitivos y seguros para sus ciudadanos, e identificar estrategias eficaces, en materia de seguridad, salud, educación, movilidad, y calidad de vida ciudadana.

\section{Antecesores de la complejidad}

\section{Antecesores de la cultura oriental}

En el siglo VI a.C. surge la filosofía, con el fin de suplantar explicaciones míticas de los fenómenos naturales por explicaciones racionales; pasando del mito al pensamiento racional. Desde esta perspectiva, la racionalidad es una facultad exclusiva de los seres humanos que incluye la coherencia teórica (conocer la realidad desde la razón) y la racionalidad práctica (ayuda a actuar desde esquemas de argumentación). Para la época no existía discrepancia entre filosofía y ciencia, pues el discernimiento se logra a través de los sentidos y ofrece una información inmediata a la 
realidad; por su parte, la razón percibe y descifra la información proporcionada por los sentidos para buscar su esencia (Figueroa et al., 2015, pp. 10-12).

Ahora bien, para evaluar la complejidad es inevitable considerar su análisis histórico. En este sentido, conviene señalar la diferencia entre antecesores y pioneros, y las diferentes teorías, que a través del tiempo son planteadas por los diferentes autores como una expresión que califica un tipo de contexto, el cual delimita concepciones de nuevo conocimiento que, de alguna forma, sobrepasan, en diversos aspectos y formas, la manera de ver la realidad, y están encaminadas a identificar el azar, la incertidumbre o el devenir (Moreno, 2003). Al realizar una línea de tiempo sobre la historia de oriente, se destacan personajes característicos, tales como: Fu-Hsi, quien a través de hexagramas, mediante la fundamentación del sistema I Ching, prescribe situaciones futuras (Wilhelm, s.f.); Lao Tsé, quien en su manual filosófico formuló una espiritualidad para equilibrar la transformación interior (Soublette, 1990); Zhuang Zhou y Fang Yizhi (como se citó en Moreno, 2003, pp. 19-20) definen el conocimiento como incierto, y cuestionan el principio de los contrarios. Contrarios, que nutrirán en occidente, siglos más tarde, a los pioneros de la complejidad.

En la tabla 1 se definen algunas alternativas propuestas por los teóricos allí mencionados, pues han sido muchos los personajes que han realizado aportes y apreciaciones significativas, donde se identifican fenómenos y eventos caracterizados por grandes desafíos, los cuales vinculan hechos inciertos para enfrentar todo aquello que genera incertidumbre, caos, incomprensión, o en cierta medida limita la posibilidad de alguna solución dentro de lo racional y lógico.

\section{Tabla 1}

Antecesores de oriente

\begin{tabular}{cl}
\hline Autor & \multicolumn{1}{c}{ Propuesta } \\
\hline & $\begin{array}{l}\text { Según la antigua tradición China, el emperador Chino Fu-Hsi inventó ocho trigramas } \\
\text { (pa-kua) que representaron el fundamento del sistema del I Ching, del cual se derivan }\end{array}$ \\
64 hexagramas.
\end{tabular}


I Ching o Libro de los cambios, escrito entre los años 2.000-1.00 a.C. es un libro oracular Chino, uno de los cinco clásicos confucianos. Significa el Libro de las Mutaciones. De procedencia taoísta y no confucionista, porque se desarrolla durante la dinastía Zhou, pero consecutivamente se retoma en la escuela de Confucio.

El I Ching describe un universo en el que la energía creadora proviene del cielo, en tanto la tierra es receptora y fecundadora de esa energía primaria. Este considera el cambio como la única realidad existente, el ser. Su filosofía supone un universo regido por el principio del cambio y la relación dialéctica entre los opuestos (Wilhelm, 2007, 2018).

Autor del libro Tao Te Ching (escrito aproximadamente hace 2.500 años), es uno de los manuales fundamentales de la filosofía y la metodología del desarrollo espiritual, el cual es profundizado en 81 enunciados espirituales (Antonov, 2008).

Lao Tsé $\quad$ En él define lo importante que es para el hombre moderno el taoísmo, pues le ayuda Siglo IV a.C. a salir del falso dilema de la existencia de Dios y definir la nueva espiritualidad que emerge de un paradigma que busca el equilibrio, rescatando la transformación interior (Soublette, 1990, pp. 6-7).

Considera que el conocimiento humano está condicionado; por ende, es incierto. A

Zhuang Zhou Zhou se le atribuyen escritos que portan la estampa de una mente brillante y original.
Su libro de 33 capítulos hace parte de uno de los textos clásicos chinos y fue

369-268 a. C premiado con el título honorífico de verdadera Escritura de Florescencia del Sur (Moreno, 2003, p. 23).

Observó el principio de la no contradicción, y lo sometió a la unidad de los contrarios,

Fang Yizhi para culminar con el acatamiento a ambos principios, cada uno en su medida.

1611-1671 Atestiguando, finalmente, que el funcionamiento de algo tiene una causalidad recursiva en vez de lineal (Moreno, 2003, p. 23).

Nota: Elaboración propia a partir de los autores referenciados.

\section{Antecesores de la cultura occidental}

Al analizar la historia del pensamiento occidental, es importante considerar cómo es percibida la realidad, y aproximarse a la visión que su cultura y entorno presentan. Bajo esta perspectiva, en la antigüedad se especulaba que admitir la condición del acontecer y el caos acarreaba ineludiblemente lo insensato y, en última instancia, la desconfianza. Para Morin (1999b), lo que afecta un paradigma, a la vez afecta la ontología, la metodología, la lógica, la epistemología, y como resultado puede modificar la práctica, la sociedad y la política (p. 51). Bajo esta premisa, la ontología de occidente se establece bajo entidades cerradas, como son la sustancia, la identidad, la causalidad, el sujeto y el objeto; dando por supuesto, entonces, que ninguna se comunica con las demás, se rechazan y se anulan.

Para examinar las diversas disyuntivas, en la tabla 2 se parte del supuesto de que la lógica de occidente era una lógica homeostática, sentenciada a mantener la proporción del discurso, el 
rechazo de la reclamación y eventual error, inquiriendo, por así decir, los desarrollos del pensamiento.

\section{Tabla 2}

Antecesores de occidente

\begin{tabular}{|c|c|c|}
\hline Autor & Año & Propuesta \\
\hline Tales de Mileto & $\begin{array}{c}625-548 \\
\text { a.C. }\end{array}$ & $\begin{array}{l}\text { Primer pensador de la filosofía griega; ofrece por primera vez una } \\
\text { explicación de la realidad basada en el carácter mítico y religioso. } \\
\text { Toma este concepto como punto de partida para alcanzar las verdades } \\
\text { más cercanas a la realidad que constituye el mundo, y enfoca sus } \\
\text { estudios hacia la naturaleza que fue la pregunta del principio o arjé, } \\
\text { del cual parte la filosofía de la escuela de Mileto (Hizmeri-Fernández, } \\
2010 \text {, p. 45; Morgan y Serrato, 2016, pp. 34-35). }\end{array}$ \\
\hline $\begin{array}{l}\text { Anaximandro } \\
\text { de Mileto }\end{array}$ & $\begin{array}{c}610-547 \\
\text { a.C }\end{array}$ & $\begin{array}{l}\text { Discípulo de Tales. Afirma que: El principio no está en los elementos } \\
\text { de la naturaleza, porque existe una lucha constante entre ellos, } \\
\text { generan destrucción y reconstrucción. Las plantas generan alimentos } \\
\text { para los animales y los humanos, y cuando estos mueren sirven de } \\
\text { abono para los árboles y plantas, creando así el ciclo percibido en el } \\
\text { ecosistema. Para el filósofo, el arjé es algo distinto a estos elementos } \\
\text { (Hizmeri-Fernández, 2010, p. 45; Morgan y Serrato, 2016, p. 35). }\end{array}$ \\
\hline Anaxímenes & $\begin{array}{c}585-525 \\
\text { a. C. }\end{array}$ & $\begin{array}{l}\text { Postula el aire como factor primigenio para el arjé, pues al estarse en } \\
\text { contacto con el frio o el aire caliente, altera la naturaleza y esto } \\
\text { transforma lo que se ve (Morgan y Serrato, 2016, p. 37). } \\
\text { Afirma que, en el aire, se presenta doble proceso: rarefacción y } \\
\text { condensación. "como nuestra alma; que es aire, nos domina y une, } \\
\text { así un aliento y un aire circunda y sujeta al mundo entero" (Gaos, } \\
\text { 1940, p. 21; Hizmeri-Fernández, 2010, p. 45). }\end{array}$ \\
\hline $\begin{array}{c}\text { Pitágoras de } \\
\text { Samos }\end{array}$ & $\begin{array}{l}580-500 \\
\text { a. C. }\end{array}$ & $\begin{array}{l}\text { Filósofo matemático. Se le atribuye el teorema de la suma de los } \\
\text { cuadrados que lleva su nombre. Su escuela se centró en solucionar } \\
\text { cuestiones del arjé colocando el número como principio. Postula el } \\
\text { número para dar sentido al pensamiento, dando a los diversos } \\
\text { números un significado (Hizmeri-Fernández, 2010, p. } 45 \text {; Morgan y } \\
\text { Serrato, 2016, p. 37). }\end{array}$ \\
\hline $\begin{array}{l}\text { Parménides de } \\
\text { Elea }\end{array}$ & $\begin{array}{c}540-470 \\
\text { a. C. }\end{array}$ & $\begin{array}{l}\text { Formula la contraparte a la propuesta de Heráclito definiendo que el } \\
\text { arjé no está en nada natural ni en el ápeiron. Su importancia radicó } \\
\text { en el ser, por ser único, inmovible e inmutable, y está por encima de } \\
\text { todo cambio y de todas las cosas, tiene existencia propia: es. El } \\
\text { primer filósofo que centra su doctrina en el estudio del Ser o Ente } \\
\text { para llegar al conocimiento. Es el referente para que filósofos } \\
\text { venideros retomen al Ser en todos sus aspectos. "la filosofía llega a } \\
\text { ser Metafísica y Ontológica", partiendo del principio que más tarde } \\
\text { fuera el Principio de Identidad: "Aquello que es, y es posible que no } \\
\text { sea" (Hizmeri-Fernández, 2010, p. } 45 \text {; Morgan y Serrato, 2016, pp. } \\
\text { 40-42). }\end{array}$ \\
\hline
\end{tabular}

Nota: Elaboración propia a partir de los autores referenciados. 


\section{Periodos filosóficos}

En cierto sentido, hay que tratar de comprender cómo se desarrolló la religión griega y los periodos filosóficos que de allí se desprenden, antes de poder entender tal proceso debidamente. No obstante, es preciso primero adquirir una idea más adecuada de la forma específica que toma el pensamiento religioso, cuando entra en la etapa de la especulación filosófica independiente (Werner, 1952, pp. 13-14). Historia filosófica, que solo puede cimentarse sobre los efectos de los filólogos que la establecen, pues ellos exteriorizan los juicios más sobresalientes de las nuevas generaciones. Sin embargo, los pensamientos filosóficos de teóricos, como los matemáticos, no son meros pensamientos particulares y anímicos, sino que conllevan a una objetividad que los sobrepasa y que dan una forma precisa al curso del pensamiento filosófico futuro.

En la tabla 3, tabla 4 y tabla 5 se realiza un esbozo de cómo la historia del mundo transita a través de períodos de razonamientos, desde la filosofía medieval, pasado por la filosofía moderna, y culminando en la filosofía contemporánea; periodos en los cuales se rotula un crecimiento paulatino de la lógica y la voluntad, desde el mundo oriental hasta el mundo grecorromano y el cristiano-germánico (Bueno, 1974, p. 20). Lo anterior, lleva a ver la filosofía bajo tres contextos diferentes: 1) como un saber acerca de las cosas, 2) como una dirección para el mundo y la vida y 3) como una forma de vida. Todas corresponden a concepciones de la inteligencia, de las cuales se ha nutrido el mundo, confluyendo a nuevas formas de intelectualidad que, aunque defectuosas y limitadas, no las descalifica, pues provienen del hombre mismo y lo que ello implica.

\section{Tabla 3}

Periodos filosóficos: Filosofía medieval

\begin{tabular}{cll}
\hline Periodo & \multicolumn{1}{c}{ Año } & \multicolumn{1}{c}{ Propuesta } \\
\hline & & La reforma de Carlos Magno afecta la cultura filosófica \\
Filosofía Medieval & Siglos II-XV & $\begin{array}{l}\text { y cultural. Se destaca Descartes como padre de la } \\
\text { Modernidad (1596-1650), iniciando la filosófica } \\
\text { moderna. Aquí se enmarca periodos como el } \\
\end{array}$ \\
& renacimiento y el humanismo (Estermann, 2001a, p.66).
\end{tabular}


La complejidad una perspectiva fflosófica
La complejidad: una perspectiva filosófica| Revista Virtual Universidad Católica del Norte, 64, 281-308

ISSN: 0124-5821 (En línea)

Época bajo la influencia de Platón y el Neoplatonismo; aquí los padres de la Iglesia buscaron argumentar racionalmente la nueva fe cristiana. Representada por Patrística notables teólogos, nombrados Padres de la Iglesia, que (120-800) salvaguardaban la enseñanza evangélica, tanto de los ataques del paganismo como de las herejías emanadas en la misma (Estermann, 2001a, p. 66; Morgan y Serrato, 2016, p. 97).

Tiempo de confusión y luchas permanentes en Europa.

Escolástica Temprana Época en la que se fortalece el Aristotelismo sobre el

(800-1200) Platonismo en occidente, a través de la filosofía árabe y judía (Estermann, 2001a, pp. 66-79).

Época de magnificencia, caracterizada por su combinación de dogmas religiosos monoteístas (judaísmo, cristianismo e islam) con métodos y

Escolástica Alta

(1200-1308) conceptos de la filosofía pagana, en especial la línea racionalista representada por Platón y Aristóteles (Estermann, 2001a, p. 66).

Critica los sistemas cerrados de la anterior, se anticipa al

Escolástica Tardía nominalismo y la ciencia. La idea existe como idea

(1308-1464) prototípica en el intelecto divino y es llevada a la existencia real por el acto creador de Dios (Estermann, 2001a, pp. 66,102).

Nota: Elaboración propia a partir de los autores referenciados.

\section{Tabla 4}

Periodos filosóficos: Filosofía moderna

\begin{tabular}{cll}
\hline Periodo & Año & \multicolumn{1}{c}{ Propuesta } \\
\hline & Se vive un cambio de paradigma (Descartes, Pascal, y \\
& Leibniz), hacia una época posmoderna donde se liberan \\
& conceptos medievales paralelamente con evoluciones \\
& científicas (Newton, Galilei, Kepler). Dios es el \\
& máximum absolutum, cristo es el médium absolutum y \\
Filosofía & el universo es el concretum máximum. El universo es \\
modernidad & Siglo XVI-XIX & infinito y no existe un centro, todo está en movimiento, \\
& incluyendo la tierra. Descartes (1596-1650) es \\
& considerado el padre de la modernidad por hacer aportes \\
& sustanciales al método matemático, el dualismo \\
& antropológico, el sujeto pensante como fundamento, la \\
& duda metódica, y la demostración de la existencia de \\
& Dios (Estermann, 2001b, pp. 66, 111-156). \\
\hline
\end{tabular}

Nota: Elaboración propia a partir de los autores referenciados. 


\section{Tabla 5}

Periodos filosóficos: Filosofía contemporánea

\begin{tabular}{|c|c|c|}
\hline Periodo & Año & Propuesta \\
\hline \multirow{5}{*}{$\begin{array}{c}\text { Filosofía } \\
\text { Contemporánea }\end{array}$} & 1850 hasta hoy & $\begin{array}{l}\text { Se dan grandes cambios: entre ellos la Revolución } \\
\text { Industrial, el evolucionismo y el antirracionalismo. Con } \\
\text { Freud, Husserl, Einstein, y Kandinsky. Para culminar con } \\
\text { estructuralismo, posmodernismo y la filosofía analítica } \\
\text { (Estermann, 2001b, p. 66). }\end{array}$ \\
\hline & $\begin{array}{c}\text { Siglo XIX (Segunda } \\
\text { Mitad) }\end{array}$ & $\begin{array}{l}\text { En } 1850 \text { se destacan representantes como: Schopenhauer, } \\
\text { Comte, Feuerbach, Kierkegaard, Bentham. La filosofía } \\
\text { contemporánea del siglo XIX está marcada por corrientes } \\
\text { como: el antirracionalismo (irracionalismo, hegelianismo } \\
\text { y marxismo), y el positivismo, neokantismo y nuevas } \\
\text { corrientes (positivismo, neokantismo, socialismo y } \\
\text { anarquismo, filosofía de vida, y pragmatismo). Todas estas } \\
\text { corrientes aportan de forma crítica a las filosofías } \\
\text { planteadas anteriormente (Estermann, 2001b, pp. 53-84). }\end{array}$ \\
\hline & Siglo XX & $\begin{array}{l}\text { Está caracterizada por tres revoluciones culturales: 1) La } \\
\text { teoría de la relatividad (Albert Einstein: la concepción del } \\
\text { espacio, el tiempo y la causalidad, donde se postulan } 4 \\
\text { dimensiones del universo). 2) El psicoanálisis (Sigmund } \\
\text { Freud: Teoría del psicoanálisis; pone de manifiesto la } \\
\text { deficiencia de una antropología dualista y el } \\
\text { descubrimiento de enfermedades psicosomáticas) y la } \\
\text { Teoría de la sexualidad, tomando como base la libido, la } \\
\text { energía sexual. 3) El arte abstracto (transforma los criterios } \\
\text { estéticos en la música, la escultura, literatura y pintura } \\
\text { (Estermann, 2001b, pp. 86-87). }\end{array}$ \\
\hline & Siglo XXI & $\begin{array}{l}\text { El conocimiento crítico es uno de los grandes logros de la } \\
\text { modernidad. Es característica por mencionarse en ella la } \\
\text { globalización de todo, relacionado por una red de } \\
\text { comunicación e intercambio. Siendo la filosofía occidental } \\
\text { un paradigma de pensamiento, sobre la dominación } \\
\text { política, económica y cultural. En él juega un papel } \\
\text { primordial la inteligencia artificial y la realidad virtual } \\
\text { (Estermann, 2001b, pp. 120-121). }\end{array}$ \\
\hline & $\begin{array}{c}\text { Filosofía } \\
\text { Latinoamericana }\end{array}$ & $\begin{array}{l}\text { Existen } 3 \text { hipótesis frente al quehacer filosófico } \\
\text { latinoamericano: 1) La organizada en universidades y en } \\
\text { círculos de revistas a lo largo del continente; 2) La que } \\
\text { proviene desde la época de la colonia y que cobra } \\
\text { importancia a partir del siglo XIX, a partir de la adaptación } \\
\text { e interculturalización del pensamiento occidental, } \\
\text { denominada por Juan Bautista Alberdi como sui géneris; y } \\
\text { 3) La de consciencia de la originalidad y originariedad de } \\
\text { un pensamiento legítimo de implantación y adaptación de } \\
\text { la tradición europea. Evolucionando de: filosofía }\end{array}$ \\
\hline
\end{tabular}


precolombina o pensamiento autóctono (S.XII-XVI), a pensamiento europeizante o implantado (1550-1980), y posteriormente a filosofía latinoamericana propiamente dicha (1960-1970) (Estermann, 2001b, pp. 124-125).

Nota: Elaboración propia a partir de los autores referenciados.

Bajo la perspectiva y parámetros de las corrientes filosóficas, consideradas en la tabla 5, se puede entender una forma en que los personajes de la época definen el problema mismo de la filosofía y el saber que perciben en ella hacia la realidad, dejando una huella perceptible y clara de la mutación misma de la realidad, apreciada en las diferentes épocas hasta su momento.

Una vez hecha esta precisión, se contempla que la cuna de la filosofía griega tuvo lugar en la costa del Asia menor, con los primeros filósofos griegos (en las costas Jónicas, ciudades helénicas de Asia Menor, entre los Siglos VI-VII). Conviene, sin embargo, añadir que los poemas de Homero fueron obras filosóficas meritorias que reflejaban la vida de los griegos, y sus pensamientos e influjos educativos de una visión pesimista de la historia, y su preocupación ética por la concepción de justicia entre los hombres (Marías, 1980, pp. 28-32; Werner, 1952, pp. 1517).

Dentro de este contexto, el paradigma antiguo occidental, establece condiciones particulares para entender la filosofía que busca el conocimiento y el entendimiento místico del hombre, concebido por los dioses, y el sumergido en el mito para expresar las formas eternas, estructuradas e inmutables del mundo, y la interpretación de ideas e idealización de lo universal y las ciencias naturales. No es indiferente, sin embargo, identificar periodos característicos en la historia de la filosofía, los cuales destacan representantes que determinan el rumbo occidental, y establecen doctrinas filosóficas que convierten el racionalismo en una doctrina de vida. Pero, la historia de la filosofía da cuenta de diversas voluntades de los individuos, en aras de encontrar la verdad a través de una argumentación coherente.

Frente a esto, algunos personajes de la filosofía griega, como Platón, definen la filosofía como un silencioso dialogo del alma consigo misma en torno al ser, afirmando la existencia de un ser inmaterial e invisible, y que es real en un sentido más profundo de lo que es el mundo material. En este mismo sentido, Kant admite que, si la elaboración de conocimientos no está orientada en una ruta segura hacia el camino de la ciencia, esta debe regresar al punto de partida e iniciar nuevamente para obtener los resultados esperados. Platón y Aristóteles, influenciados por 
Sócrates, buscaron la verdad en la filosofía antropológica y ética, mediante la utilización del método del dialogo, la mayéutica y la nesciencia, con el objetivo de cuestionar frente a la doctrina filosófica, la justicia y la vida. En tanto, Parménides, aduce que la filosofía no está en las cosas, sino fuera de ellas: en las ideas. Y estas últimas son entes metafísicos que encierran el verdadero ser de las cosas (Marías, 1980, pp. 24-67).

El surgimiento del cristianismo trae consigo una nueva concepción que da sentido a la filosofía moderna y a la existencia del universo y de los individuos. Aduciendo que lo que se opone al ser, es el no ser. En efecto, se plantea así un cambio de perspectiva desde dos sentidos diferentes: el ser de Dios y el del mundo. A este propósito, San Agustín (354-430 d.C.) se convierte en el más importante de los Padres de la Iglesia latina, pues recoge una serie de doctrinas helénicas, adaptando aportaciones de los griegos a las necesidades filosóficas del dogma cristiano (Marías, 1980, pp. 101-132).

En su acepción estricta, la idea de filosofía limita la amplitud y con ella la interpretación que se ajusta a la acepción. Ahora, la filosofía es, ante todo, como las matemáticas, una institución de tradición helénica, una formación cultural del área de difusión helénica. Esta tradición comporta, entre otras cosas, un vocabulario característico, que puede sintetizarse en un amplio conjunto de nombres que designan indudables ideas (Bueno, 1974, pp. 12-13).

\section{Pioneros de la complejidad}

Todo lo planteado anteriormente puede contrastarse con la concepción lineal que corresponde a planteamientos que condicionan el conocimiento humano a un Ser supremo y les permite adoptar concepciones diversas de explicar el origen de las cosas, pero que los sumerge en un paradigma que cuestiona su conocimiento e interioridad para buscar un equilibrio y dar respuesta a fenómenos que no podrían explicar. Otra conexión con el pensamiento complejo y la complejidad misma.

Como se muestra en la tabla 6 y la tabla 7, tales condiciones generan cuestionamientos que llevan a deducir a estos pioneros de la complejidad una línea eje que parte desde 1948, con la teoría cibernética y las maquinas artificiales, hasta la teoría general de sistemas, y que traspasa círculos culturales diferentes del helenismo y el racionalismo, exponiendo con una nueva visión, surgida 
La complejidadr una perspectiva fflosófica
La complejidad: una perspectiva filosófica| Revista Virtual Universidad Católica del Norte, 64, 281-308

ISSN: 0124-5821 (En línea)

del enriquecimiento de los filósofos que le anteceden, el concepto de materialismo histórico, hasta la adopción de sistemas de transformación y estructuración de algoritmos que ayuden a la comprensión y adaptación de metodologías, las cuales definan las dinámicas de las organizaciones contemporáneas que, alimentadas de la complejidad, van generando tanto problemáticas como soluciones a las necesidades productivas y sociales, hasta la década de los años 70 .

De aquí que se utilicen conceptos existenciales nativos y cuasi mágicos que recogen el hecho de simultaneidad y magnitud histórica y no empírica para explicar las crisis de la humanidad de forma mística y sustancial. En este marco, considera similitudes acontecidas en el viejo mundo y en varios periodos de tiempo, que son, en sí mismas, complejidad no declarada (Bueno, 1974, pp. 36-42).

\section{Tabla 6}

Pioneros de la Complejidad (1948-1950)

\begin{tabular}{|c|c|c|}
\hline $\begin{array}{l}\text { Año de } \\
\text { formulación } \\
\text { de la teoría }\end{array}$ & Autor & Propuesta \\
\hline $1948-1950$ & Norbert Wiener & $\begin{array}{l}\text { Introdujo al lenguaje científico el concepto de cibernética. Parte } \\
\text { del hecho del concepto de mecánica estadística consistente en } \\
\text { resolver una contingencia compleja en una secuencia infinita de } \\
\text { múltiples contingencias especiales, cada una con probabilidad } \\
\text { conocida, de esto parte el hecho de la paradoja que la suma de } \\
\text { la probabilidad cero deriva de la probabilidad uno. Desarrolló } \\
\text { un lenguaje y técnicas para establecer los límites de las } \\
\text { comunicaciones entre los individuos (Wiener, 1948, pp. 24-46). }\end{array}$ \\
\hline \multirow[t]{2}{*}{1950} & $\begin{array}{c}\text { Janos Von } \\
\text { Neumann y Alan } \\
\text { Turing }\end{array}$ & $\begin{array}{l}\text { Plantearon el problema de la diferencia entre las máquinas } \\
\text { artificiales (simplemente organizadas) y las máquinas vivas } \\
\text { (auto-organizadoras) (Turing, 1950, p. 14); realizaron aportes } \\
\text { substanciales a la cibernética, al admitir la idea de } \\
\text { autoorganización, y aseverar que la máquina analítica era un } \\
\text { computador universal digital (Neumann y Turing, como se citó } \\
\text { en Morin, 1999b, p. } 31 \text { y Moreno, 2003, p. 24). }\end{array}$ \\
\hline & $\begin{array}{l}\text { Ludwig Von } \\
\text { Bertalanffy }\end{array}$ & $\begin{array}{l}\text { Formuló la Teoría general de sistemas; disciplina que } \\
\text { manifiesta y deriva de aquellos principios que son válidos para } \\
\text { los sistemas en general. Esta, estudia los principios aplicables a } \\
\text { los sistemas en cualquier nivel, en todos los campos de la } \\
\text { investigación (Bertalanffy, 1968, p. 32). }\end{array}$ \\
\hline
\end{tabular}

Nota: Elaboración propia a partir de los autores referenciados. 


\section{Tabla 7}

Pioneros de la Complejidad (1999-2008)

\begin{tabular}{|c|c|c|}
\hline $\begin{array}{l}\text { Año de } \\
\text { formulación } \\
\text { de la teoría }\end{array}$ & Autor & Propuesta \\
\hline \multirow{6}{*}{ 1999-2008 } & $\begin{array}{l}\text { Heinz Von Foerster } \\
\text { (s.f.) }\end{array}$ & $\begin{array}{l}\text { Propuso el principio de orden por ruido que refuerza la } \\
\text { deducción de la creación de un orden a partir del desorden. } \\
\text { Plantea el principio de orden por ruido, reforzando la deducción } \\
\text { de la creación de un orden a partir del desorden (Foerster, como } \\
\text { se citó en Morin, 1999a); mencionado en Moreno }(2003 \text {, p. 25) } \\
\text { y Osorio et al. }(2008, \text { p. } 33) \text {. }\end{array}$ \\
\hline & Henri Atlan (s.f.) & $\begin{array}{l}\text { Concibió la teoría del azar organizador, formulando el } \\
\text { nacimiento del universo y de la vida a partir de la dialógica } \\
\text { orden/desorden/organización (Atlan, como se citó en Moreno, } \\
2003, \text { p. 25). }\end{array}$ \\
\hline & $\begin{array}{c}\text { Magoroh Maruyama } \\
\text { (1929) }\end{array}$ & $\begin{array}{l}\text { Retoma el concepto de cibernética de Wiener (1958): la } \\
\text { autodirección, y autorregulación, al cambiar de estado, en tanto } \\
\text { mantenía su forma original en el sistema (morfostasis). } \\
\text { Permanecía en equilibrio a través de un intercambio } \\
\text { homeostático con el entorno; explicando de esta forma el } \\
\text { comportamiento de las maquinas, mas no del organismo vivo. } \\
\text { Maruyama explicó la retroalimentación positiva, al permitir al } \\
\text { sistema adoptar una nueva organización, transformación o } \\
\text { cambio (morfogénesis) (Maruyama, como se citó en Moreno } \\
(2003, \text { p. 24), Osorio et al. (2008, p. 31), y Wiener (1958). }\end{array}$ \\
\hline & $\begin{array}{c}\text { Claude Shannon } \\
\text { (1948) }\end{array}$ & $\begin{array}{l}\text { Usó conceptos de la mecánica estadística para asegurar que las } \\
\text { posibles distorsiones por interferencia de una fuente de ruido } \\
\text { pudieran ser corregidas por el receptor. } \\
\text { Al lado de Warren Weaver desarrollo la teoría matemática de la } \\
\text { información (mencionados en Moreno }(2003 \text {, p. } 24) \text {, Osorio et } \\
\text { al. }(2008 \text {, p. } 30) \text { y Shannon \& Weaver }(1948, \text { p. } 31) \text {. }\end{array}$ \\
\hline & $\begin{array}{l}\text { Humberto Maturana } \\
\text { y Francisco Varela } \\
\text { (1990) }\end{array}$ & $\begin{array}{l}\text { Proponen conceptos desde la biología para explicar la idea de } \\
\text { autoorganización, tales como: "autopoiesis" y "acoplamiento } \\
\text { estructural". Al mismo tiempo, realizaron aportes para la } \\
\text { comprensión del problema epistemológico de la reflexividad y } \\
\text { del conocimiento de segundo orden (como se citó en Moreno, } \\
2003 \text {, p. } 25 \text {, y Osorio et al., 2008, p. 59). }\end{array}$ \\
\hline & $\begin{array}{c}\text { llya Prigogine } \\
\text { (1997) }\end{array}$ & $\begin{array}{l}\text { Introdujo la idea de organización a partir del desorden desde la } \\
\text { termodinámica, la bioquímica y la microbiología. Para analizar } \\
\text { la dinámica y la termodinámica los únicos procesos que deberían } \\
\text { existir son los irreversibles siendo este un obstáculo entrópico } \\
\text { de las condiciones iniciales, pues la irreversibilidad no es una } \\
\text { propiedad universal y la complejidad debe estar definida en } \\
\text { términos físicos y no en términos de conocimiento (como se citó } \\
\text { en Moreno, 2003, p. } 25 \text {, Osorio et al., } 2008 \text { p. } 51 \text {, y Prigogine y } \\
\text { Stengers, } 1997, \text { p. 232). }\end{array}$ \\
\hline
\end{tabular}


Nota: Elaboración propia a partir de los autores referenciados.

\section{Teorías que aportan a la complejidad}

A partir del siglo XX la actividad filosófica latinoamericana sufre una transformación en sus enfoques filosóficos, determinados y surgidos en la complejidad misma del momento citado por Estermann (2001a), y por el reflejo e influencia de la filosofía europea y norteamericana, que desde los contextos problémicos y específicos del pensamiento de la época, dejan permear que esta ha sido sui géneris, concebida a partir de la dependencia socio-económica y cultural, que la llevaron a una desincronización política, tecnológica, informática y económica. Es así como tuvo que enfrentarse a tres etapas cruciales para definir su propia filosofía: 1) su pensamiento autóctono, 2) el pensamiento implantado desde la colonización, y 3) la filosofía de liberación (Estermann, 2001a, pp. 124-134).

Este corto recorrido por el pensamiento del hombre y la filosofía, a través del tiempo, permite establecer una conexión entre el antiguo oriente y occidente, pasando obligadamente por la antigua Grecia; conexión forzosa de la filosofía, la metafísica, el racionalismo, la complejidad, la dialéctica y el pensamiento complejo, asimilados a los conceptos de ciudades inteligentes, sus problemáticas y formas de aplicarlos a la solución que ellas pretenden. Para Morin (1999a), la complejidad es un tejido, complexus de constituyentes heterogéneos inseparablemente asociados, donde se presenta la paradoja de lo uno y lo múltiple. En este sentido, afirma:

El concepto de complejidad se ha formado, agrandado, extendido sus ramificaciones, pasando de la periferia al centro de mí meta, devino un macro-concepto, lugar crucial de interrogantes, ligando en sí mismo, de allí en más, al nudo gordiano del problema de las relaciones entre lo empírico, lo lógico, y lo racional. (p. 24)

Visto de desde aquí, y no siendo la complejidad un punto de partida o de llegada, sino una opción intermedia no terminada y en exploración (Morin, 2008), se puede admitir que los grandes problemas no resueltos por la humanidad, en los últimos 70 años, tal y como la complejidad, no han tenido un punto de llegada, y aunque tuvieron un punto de partida, este estaba permeado por el paradigma dominante; y lo estaba porque todo el legado de conocimiento recibido, que fundamentó todo el acervo de directrices gubernamentales, educativas, sociales, y religiosas en 
general, se convirtió, por defecto, en un punto de llegada caótico y un poco endeble, propio de lo inacabado como la complejidad misma, e incapaz de solucionar problemáticas, como las que enfrentan las grandes ciudades en todo el universo hoy.

Ahora bien, el ser humano, en su complejidad, es el centro en los enfoques del paradigma emergente, lo que le permite ser redimido y programado, para resarcir en cierta medida esa deuda de siglos en los pueblos del Sur (Castillo, 1997; Morin, 2008; Souza Santos, 2010), argumento denominado como "Paradigma Perdido"; en este método en gestación se reformula el concepto de hombre racional y pragmático que guía el pensamiento y las ciencias, válidamente aceptadas, enfrentándose ahora a nuevas visiones: ese conocimiento científico vs el sentido común, sumado a la naturaleza misma y la persona humana; al hombre como ser.

La industrialización de las ciencias, tanto en el norte como el viejo continente, estratificó y deslegitimó aún más todo el enfoque y la comunidad científica, junto con sus paradigmas científicos y la denominada ecología de saberes, donde se abordan saberes no científicos ancestrales que sustentan lo sagrado (Souza Santos, 2010; Souza, como se citó en Aragón Andrade, 2011). Esto quiere decir que se eliminó y se alejó a las demás corrientes de pensamiento, ajenas o no alineadas con los dos grandes bloques de la política y la industria en el mundo: este y oeste. Esto es, por decir así, el nacimiento de toda esta indisposición social, insatisfacción, desánimo científico y de incertidumbre sobre la sustentabilidad, el paradigma emergente.

La complejidad tiene que ver con la aparición del cambio, del devenir, la constitución de nuevos órdenes, donde el mismo devenir se convierte en principio constitutivo y explicativo. Es entonces cuando el pensamiento complejo abarca corrientes filosóficas y pensamientos surgidos a través de las diferentes teorías, las cuales varían significativamente según el área del conocimiento considerada (Moreno, 2003, p. 22). En la tabla 8, cuando se analiza lo anterior, Lorenz (1963, como se citó en Gleick, 1987), Prigogine y Stengers (1997), Shannon (1948), Morin (s.f.), Bertalanffy (1968), y García (1994), realizan aportes que, desde las teorías del caos, la algorítmica de la información, la de la simplicidad, y la teoría general de sistemas, contribuyen a analizar el paradigma de la complejidad (Tablas 8,9 y 10).

Cuando se analiza lo anterior, en la tabla 8 se muestra como Lorenz (1963, citado en Gleick, 1987), Prigogine y Stengers (1997) y Shannon (1948) realizan aportes interesantes desde la teoría del caos. Ahora bien, en la tabla 9, Morin (s.f.), Bertalanffy (1968), y García (1994) realizan 
La complejidadr una perspectiva fflosófica
La complejidad: una perspectiva filosófica| Revista Virtual Universidad Católica del Norte, 64, 281-308

ISSN: 0124-5821 (En línea)

consideraciones que validan la justificación de los fenómenos del universo y el paradigma que ello genera para los diferentes autores, al definir las teorías algorítmica de la información y la de la simplicidad, llevando esto, finalmente, a mostrar en la tabla 10, como Bertalanffy (1968), García (1994) y Moreno (2003), con la teoría general de sistemas y la interacción de los diferentes subsistemas que lo componen, contribuyen a analizar el paradigma de la complejidad.

\section{Tabla 8}

Teorías que plantean la Complejidad: "Teoría del Caos"

\begin{tabular}{|c|c|c|}
\hline Teoría & Autores & Propuesta \\
\hline \multirow{3}{*}{ Teoría del Caos } & $\begin{array}{l}\text { Edward } \\
\text { Norton } \\
\text { Lorenz } \\
\text { (1963, como } \\
\text { se citó en } \\
\text { Gleick, } \\
1987,) \\
\end{array}$ & $\begin{array}{l}\text { Diseñó un modelo de condiciones meteorológicas consistente en } \\
\text { tres ecuaciones no-lineales vinculadas. Manifestó que las } \\
\text { soluciones de estas ecuaciones eran extremadamente sensibles a } \\
\text { las condiciones iniciales. Desde el mismo punto de origen, dos } \\
\text { trayectorias se desarrollan de modo completamente distinto, } \\
\text { haciendo imposible toda predicción a largo plazo (Lorenz, como } \\
\text { se citó en Gleick, 1987, pp. 20-24). }\end{array}$ \\
\hline & $\begin{array}{l}\text { Almarza } \\
\text { Rísquez, F. } \\
\text { (s.f.). }\end{array}$ & $\begin{array}{l}\text { Estudia sistemas dinámicos complejos, en los que la aparición de } \\
\text { problemas y aberraciones frecuentes intrigaba a los estudiosos y } \\
\text { que ahora son vistas con carácter de existencia legítima. No es } \\
\text { antirracionalista; pretende acrecentar los alcances del } \\
\text { conocimiento, liberándola de sus restricciones empíricas actuales. }\end{array}$ \\
\hline & $\begin{array}{l}\text { Prigogine y } \\
\text { Stengers } \\
(1997, \text { p. } 10)\end{array}$ & $\begin{array}{l}\text { Mostró que las estructuras complejas podrían resultar siendo las } \\
\text { más simples, afirmo que el no equilibro es fuente de orden, de } \\
\text { coherencia; y entre las unidades surgen correlaciones. A este } \\
\text { propósito, era de crucial importancia lo no-lineal para describir los } \\
\text { sistemas auto-organizadores, siendo este el punto de partida para } \\
\text { la teoría laser. }\end{array}$ \\
\hline
\end{tabular}

Nota: Elaboración propia a partir de los autores referenciados.

Tabla 9

Teorías que plantean la Complejidad: "Teoría de Algorítmica y Teoría de simplicidad"

\begin{tabular}{ccl}
\hline Teoría & Autores & \multicolumn{1}{c}{ Propuesta } \\
\hline & $\begin{array}{l}\text { Basada en la complejidad de Kolmogorov, para la } \\
\text { determinación del contenido de la información, definido este } \\
\text { por una expresión isomorfa con la entropía negativa de la } \\
\text { Teoría Algorítmica } \\
\text { de la información }\end{array}$ & Shannon (1948) \\
\cline { 2 - 3 } & Abramson & $\begin{array}{l}\text { Su objetivo es desarrollar correspondencia, entre el lenguaje de } \\
\text { las matemáticas para expresar la teoría de la información. }\end{array}$ \\
\hline
\end{tabular}


(1981, pp. 4-

14)

Morin

simplicidad

(s.f., p. 2)

Entendiendo el problema de la complejidad y sabiendo que el paradigma de simplicidad está compuesto por relaciones lógicas entre nociones maestras, claves y principios que rigen los discursos que acatan instintivamente a su gobierno. Tal paradigma, pone orden en el universo y persigue al desorden. En este sentido, la complejidad de la relación orden-desordenorganización fluye cuando se comprueba que fenómenos desordenados, son necesarios en ciertos contextos, para provocar fenómenos organizados, favoreciendo estos el incremento del orden.

Nota: Elaboración propia a partir de los autores referenciados.

Tabla 10

Teorías que plantean la Complejidad: "Teoría de Sistemas"

\begin{tabular}{|c|c|c|}
\hline Teoría & Autores & Propuesta \\
\hline \multirow{3}{*}{ Teoría de sistemas } & $\begin{array}{l}\text { Bertalanffy } \\
\quad(1968)\end{array}$ & $\begin{array}{l}\text { Es el reemplazo de la concepción, todo/partes por la concepción } \\
\text { sistema/entorno. Parte y todo son categorías que resultan } \\
\text { insuficientes en una naturaleza inorgánica. "Existen modelos, } \\
\text { principios y leyes aplicables a sistemas generalizados o a sus } \\
\text { subclases, sin importar su particular género, la naturaleza de sus } \\
\text { elementos componentes y las relaciones o fuerzas, que imperen } \\
\text { entre ellos. Parece legítimo pedir una teoría no ya de sistemas de } \\
\text { clase más o menos especial, sino de principios universales } \\
\text { aplicables a los sistemas en general" (p. 32). }\end{array}$ \\
\hline & $\begin{array}{c}\text { Moreno } \\
\text { (2003, p. 26) }\end{array}$ & $\begin{array}{l}\text { El entorno deja de ser un componente condicionante de la } \\
\text { construcción del sistema pasando a ser un factor constituyente de } \\
\text { ella. }\end{array}$ \\
\hline & $\begin{array}{c}\text { García } \\
(1994, \text { p. 93) }\end{array}$ & $\begin{array}{l}\text { Los sistemas complejos están constituidos por elementos } \\
\text { heterogéneos en interacción, donde sus subsistemas pertenecen a } \\
\text { los dominios materiales de diversas disciplinas. En este proceso, } \\
\text { la adición aislada de sus componentes explica su totalidad. Sus } \\
\text { principales características: 1) la carencia de límites bien } \\
\text { definidos; 2) son abiertos (mantienen interacciones con su } \\
\text { medio); 3) presentan la impredecibilidad de su aparición desde un } \\
\text { estado previo; 4) el todo es más que la suma de sus partes; 5) } \\
\text { tienen intercambios con el entorno; 6) son fuente de una } \\
\text { problemática no reducible a fenómenos que pertenezcan al } \\
\text { dominio exclusivo de una disciplina. }\end{array}$ \\
\hline
\end{tabular}

Nota: Elaboración propia a partir de los autores referenciados. 
La complejidad es un modo de pensamiento que vincula tanto el orden, lo universal y lo usual, como el caos, lo específico y el devenir. Frente a esto, las diversas formas de conocimiento otorgan una representación de discernimiento que admite ilustraciones de las ciencias naturales, para interpretar los sucesos de la naturaleza, pero concluye al suponer que siempre queda pendiente algo más por expresarse. Al explorar el campo de la complejidad se indaga, en múltiples disciplinas, que en fin de cuentas lo que permite es la inserción en un tipo de pensamiento, en aras de sustentar lo complejo de una forma más simple posible y aplicable, pero no finita ni terminada.

\section{Ciudades inteligentes}

Ciudad inteligente es un concepto que por su reciente aparición aún se halla en construcción y desarrollo, como parte de las alternativas de solución a las nuevas problemáticas y retos del siglo XXI, no solucionadas aún con las herramientas, enfoques administrativos de lo público y sistemas tradicionales de solución, gubernamentales y privados. En este sentido, las ciudades inteligentes proporcionan oportunidades en las distintas áreas de actuación, relacionadas con las personas, la movilidad, el empleo, el urbanismo y vivienda, el gobierno y las administraciones, las energías renovables y la sostenibilidad energética, medioambiental, económica y social, para mejorar la calidad de vida de las personas y favorecer la actividad empresarial y laboral (Grupo Interplataformas de Ciudades Inteligentes [GICI], 2016, p. 13; Maestre, 2017, p. 26).

La literatura científica publicada hasta el momento no ha especificado de forma particular cual podría ser la definición más apropiada para lo que hoy se conoce como una ciudad inteligente, en parte por lo inacabado del concepto y su creciente ramificación, en búsqueda de soluciones acordes a los nuevos retos. Dentro de este componente, se pueden rescatar algunas definiciones que ayudan a entender un poco su significado y la necesaria e infaltable contextualización de todas ellas, como la complejidad misma que las guía, y las corrientes filosóficas que las plantean y les dan origen.

Frente a esto, Abad et al. (2018) afirman que una ciudad es inteligente cuando al menos es capaz de desarrollar una iniciativa que aborde una o más de las variables consideradas decisivas en la solución de las problemáticas de estas ciudades: economía inteligente, personas inteligentes, 
movilidad inteligente, entorno inteligente, gobierno inteligente y forma de vida inteligente (Abad et al., 2018, p. 29). Y Bouskela et al. (2016) definen la ciudad inteligente como aquella que coloca a las personas en el centro del desarrollo, incorporando las TIC en la gestión urbana y usando estos elementos como herramientas para estimular la información de un gobierno eficiente, que incluya procesos de planificación colaborativa y ciudadana (p. 16).

Hasta aquí es deducible que las problemáticas no resueltas en estas ciudades y otros escenarios poblacionales que evolucionan hacia esta modalidad de ciudad no han logrado aportar soluciones lógicas racionales y efectivas, desde la óptica filosófica y de conocimiento del paradigma dominante (Morin, 2008). Es ahí donde el pensamiento complejo aporta oportunidades de solución no convencionales, como propuesta viable y segura para los nuevos retos no resueltos.

Se contextualiza y sincroniza, de esta manera, la utilidad de las posturas de la complejidad y multidisciplinariedad, que confluyen como dinamizadores de alternativas de solución viables, reales y esencialmente efectivas (Sotolongo Codina y Delgado Díaz, 2006).

\section{Discusión}

Centrarse y centrar las ciencias en lo observable y medible, es corto y reduccionista. Esta visión no sería posible si no se parte de las ideas, manifestando lo esencialmente humano a las cosas, porque esa es una forma reduccionista que sobrepone, sobre el ser humano, las mediciones como único parámetro para el rigor científico, desconoce las cualidades de la cosa misma, eliminado el ascenso a conocimientos más profundos. Examinar brevemente que "a medida que las ciencias naturales se aproximan a las ciencias sociales, estas se aproximan a las humanidades" (Morín, 2008), y son consideraciones que fundamentan el paradigma emergente, aún obstaculizan la cientificidad de las ciencias sociales, inclusive sabiendo del papel de estas, en favor del desarrollo de las ciencias naturales (Morín, 2008).

Del mismo modo, la simulación de una organización social da cuenta de cambios de un sistema en acción, en donde los procesos dinámicos en el tiempo son regulados y estabilizados. Por tanto, las innovaciones técnico-metodológicas de las ciencias complejas producen nuevos enfoques hacia el conocimiento continuo hegemónico y dominante de la ciencia (Maldonado, 2009, pp. 9-14; Rodríguez Zoya y Aguirre, 2011). Ofrecen, igualmente, herramientas en el 
abordaje de fenómenos complejos (auto-organización), utilizando metodologías de modelización y simulación computacional de sistemas complejos y técnicas basadas en modelos de agentes, que ayudarían a perfilar y mejorar los actuales modelos de ciudad inteligente, fundamentados y un poco asimilados a ciudades digitales, antecesoras de la ciudad inteligente, pero igualmente reduccionistas, si persisten en ignorar que lo digital es un paso en el proceso del nuevo paradigma emergente del pensamiento complejo y, simplemente, un instrumento más en la nueva construcción pretendida, no un fin.

La literatura existente ha encontrado una fuerte relación entre las estrategias que utilizan las ciudades para salir de sus crisis económicas, sociales y ambientales, y el paradigma de conocimiento y creatividad inteligente de sus ciudadanos, para utilizar tecnologías de desarrollo urbano que les permita implementar doctrinas acordes a los objetivos de desarrollo sostenible. En esta medida es interesante observar que, en todos los casos de estudio, se observa que las ciudades comparten espacios que promueven la capacidad de innovar en sus territorios, convirtiendo a sus moradores en individuos creativos capaces de sortear actividades que les permitan la adaptación de conocimiento para administrar y resolver problemas que ayuden a mejorar sus actividades y funciones urbanas.

Sumado a lo anterior, el crecimiento de las ciudades representa un gran reto para los gobiernos actuales, pues este implica una amplia correlación entre su crecimiento económico y su gestión urbanística, acorde o no con los planes de ordenamiento territorial (POT), trayendo consigo múltiples problemáticas. En cierta forma, esto genera altos índices de pobreza, pues sus ciudadanos son conscientes de lo que el mal manejo de los espacios urbanísticos puede llegar a ocasionar. Consecuencia de ello, es que se deben formular políticas públicas para educar a sus ciudadanos y disminuir la brecha de ignorancia que muchas veces genera altos índices de inequidad urbana, y lleva a una lenta y decreciente inequidad social, que sumado al incremento de la delincuencia y un deficiente acceso a servicios públicos domiciliarios, trae consigo un retroceso económico que no permite a los territorios manejar y tener claridad de cuáles serían sus prioridades, para avanzar en este camino de convertirse en el mediano plazo en una ciudad inteligente.

La complejidad permite visualizar las manifestaciones anómalas innatas, en algunos de los territorios, que no admiten sortear ni abreviarse, al considerar el desorden y las problemáticas sociales propias de las ciudades, bien que estén o no en vía de ser inteligentes; tal situación puede 
La complejidadk una perspectiva fflosófica
La complejidad: una perspectiva filosófica| Revista Virtual Universidad Católica del Norte, 64, 281-308

ISSN: 0124-5821 (En línea)

incluso aceptarse como proceso. No obstante, por el contrario, es que debe procurar concebirse hasta donde sea permitido. El estudio de la complejidad puede proporcionar una perspectiva de la dinámica urbana más consciente y escalonada en el tiempo que otras orientaciones más holísticas e inmutables. En esta medida, nos ayuda a entender de forma más simple los diferentes contextos y escenarios que se presentan en estos entornos urbanos, para definir, a partir de allí, soluciones que posibiliten explorar novedosas y nativas formas desde la complejidad y el territorio mismo, que lleven a generar mejoramiento en la calidad de vida de sus ciudadanos.

\section{Conclusiones}

Abordar desde la complejidad y las teorías filosóficas recorridas: filosofía medieval, moderna y contemporánea, una técnica que se adapte y permita dar soluciones de fondo a las problemáticas planteadas, no es precisamente fácil y no es lo que se pretende. Pero si se permite y puede establecer relaciones entre ellas que aporten en la búsqueda de alternativas de solución, objeto sí, estas últimas, de la complejidad misma y este recorrido. En este punto, desde el "paradigma dominante", asume que los fenómenos antroposociales no obedecen a principios inteligibles poco complejos de aquellos fenómenos naturales que los generan.

La ciencia no es el estudio del universo simple, es una simplificación heurística necesaria para ver ciertas leyes. Aún los individuos permanecen ciegos al problema de la complejidad, y de algún modo se aferran a los conocimientos que durante siglos proporcionó una comunidad científica reconocida y autorizada, pero que endiosada en la novedad y rigurosidad de sus descubrimientos y linealidades, no se cuestionó, no se criticó ni dio vía a las opciones, de algún modo caóticas, asimétricas y desarmónicas, que ahora precisamente se yerguen como alternativas defensoras de lo social, de lo humano y de todo lo que las ciencias naturales no han podido explicar, corregir ni reinventar.

Es fácil comprender, entonces, porqué algunos autores omiten estas opciones y prefieren pasar de largo, pero esa negación a la verdad hace parte de la ignorancia en el cual se encuentran sumidas las personas. Se tiene que aprender que se está siempre en la prehistoria del espíritu humano y contextualizando el pensamiento complejo, aplicado a nuevas búsquedas de 
conocimiento, aperturas y perspectivas sobre lo establecido, para dar cabida a la complejidad y la riqueza de posibilidades que induce, sin deslegitimar del todo lo hasta ahora realizado.

Debe quedar claro que la intención es realizar un comparativo analógico de las afirmaciones que los autores observan y deducen sobre los temas de la complejidad y la ruptura que sus observaciones plantean a las teorías y postulados de las ciencias naturales convencionales y al paradigma dominante que las respalda. Habría que abordar seguidamente la posibilidad de aplicar tales posibilidades e interpretaciones investigativas y prácticas del "paradigma emergente", a las problemáticas de la sociedad de hoy y la alternativa de las ciudades inteligentes.

Las diversas apreciaciones realizadas por los investigadores citados dan certeza y vida al paradigma emergente, pero validan a la vez que esta no es la única visión. Por el contrario, abren y posibilitan nuevos enfoques de auto sostenibilidad; misma que se plantean en las variables más comúnmente tenidas en cuenta en las smart cities, soportados bajo la guía metodológica del "paradigma emergente" y la interpolaridad con el pensamiento complejo, en la nueva dinámica de las ciudades inteligentes, para trabajar en ellas con entornos y parámetros heterogéneos, multifuncionales y en diferentes niveles de abstracción.

El artículo es, en gran medida, un insumo para la comprensión de las dinámicas de las ciudades inteligentes; en este sentido, sirve como punto de partida para futuras líneas de investigación enfocadas a la comprensión de las problemáticas planteadas y observables para las ciudades inteligentes, y las futuras que puedan derivarse de ellas o no. La realidad es dinámica y creciente en soluciones y nuevas problemáticas, que al igual que las teorías y complejidad misma, serán siempre encabadas y con nuevos retos, no todos visibles ahora.

\section{Referencias}

Abad, F., Bermejo, M. G., Briz, L., Campillo, Z., Díaz, R. F., García, T., y Vega, D. D. (2018). Informe sobre La tendencia inteligente de las ciudades en España: Grupo de Smart Cities/Smart Regions. Asociación Española de Ingenieros de Telecomunicaciones. https://www.coit.es/sites/default/files/informes/pdf/2018-01$\underline{30 \_ \text {coit_grupo_scsr_informe_tendencia_smart_version_final.pdf }}$ 
Abramson, N. (1981). teoría de la Información y la Codificación (5 ed.). McGRAWHILL.https://www.academia.edu/19621469/Teoria_de_la_Informacion_y_codificacion_ Norman_Abramson_ebook_spanish

Almarza Rísquez, F. (s.f.). La Teoría del Caos. Modelo de interpretación epistémica e instrumento de solución: reconciliación entre ciencias y humanidades. https://pensamientocomplejo.org/mdocs-posts/almarza-risquez-f-la-teoria-del-caosmodelo-de-interpretacion-epistemica-e-instrumento-de-solucion-reconciliacion-entreciencias-y-humanidades/

Antonov, V. (2008). Lao Tsé Tao Te Ching. Lulu. https://docplayer.es/421993-Lao-tse-edicionpor-vladimir-antonov-traducido-al-espanol-por-anton-teplyy.html

Aragón Andrade, O. (2011). Una epistemología del Sur: La reinvención del conocimiento y la emancipación social. Alteridades, 21(41), 181-184. http://www.scielo.org.mx/scielo.php?script=sci_arttext\&pid=S0188$\underline{70172011000100017 \& \ln g=e s \& t \ln ==e s}$

Bertalanffy, L. V. (1968). Teoría General de Los Sistemas: Fundamentos, desarrollo, aplicaciones (J. Almela, Trad.). Fondo de Cultura Económica. https://cienciasyparadigmas.files.wordpress.com/2012/06/teoria-general-de-los-sistemas_-fundamentos-desarrollo-aplicacionesludwig-von-bertalanffy.pdf

Bouskela, M., Casseb, M., Bassi, S., De Luca, C., y Facchina, M. (2016). La Ruta Hacia las Smart Cities: Migrando de una gestión tradicional a la ciudad inteligente. BID. https://publications.iadb.org/bitstream/handle/11319/7743/La-ruta-hacia-las-smart-citiesMigrando-de-una-gestion-tradicional-a-la-ciudad-inteligente.pdf

Bueno, G. (1974). La Metafísica Presocrática. Historia de la Filosofía. Pentalfa Ediciones. https://efimeroescombrera.wordpress.com/2010/06/18/libro-g-bueno-la-metafisicapresocratical

Caragliu, A., Del Bo, Ch. \& Nijkamp P. (2011). Smart cities in Europe. Journal of Urban Technology, 18(2), 65-82. https://doi.org/10.1080/10630732.2011.601117

Castillo, J. (1997). El paradigma perdido de la interdisciplinariedad: volver a los clásicos. Política y Sociedad, 26, 143-155. https://core.ac.uk/download/pdf/38819343.pdf 
Estermann, J. (2001a). Curso Integral de Filosofía desde América Latina 2. Historia de la Filosofía Primera Parte. Tomo II. Ediciones Abya-Yala.

Estermann, J. (2001b). Curso Integral de Filosofía desde América Latina 3. Historia de la Filosofía Segunda Parte. Tomo III. Ediciones Abya-Yala.

Figueroa, P., Martínez C. J., López B. M., y Lozano, M. (2015). BL Filosofía 1 Batxilergoa. Euskera. https://www.mheducation.es/bl-filosofia-1-batxilergoa-euskera-libro-digital9788448607999-spain

Gaos, J. (1940). Antología filosófica. La filosofía griega. La Casa de España en México. http://www.cervantesvirtual.com/bib/extras_autor/00002616/hipertextos/pdf/af/gaosaf.P $\underline{\mathrm{DF}}$

García, R. (1994). Interdisciplinariedad y sistemas complejos. En E. Leff (Comp.), Ciencias sociales y formación ambiental (pp. 85-124). Gedisa.

Gleick, J. (1987). Chaos: Making a New Science. Viking Penguin.

Grupo Interplataformas de Ciudades Inteligentes -GICI-. (2016). Smart Cities: Documento de Visión $a$ 2030. GICI. http://gici.eu/sites/default/files/gici/public/contentfiles/pages/Presentacion\%20resumen\%20GICI_esp.pdf

Hizmeri-Fernández, J. (2010). Paradigma de la Complejidad, Educación, Curriculum y Praxis docente (Tesis de Maestría). Universidad del Bio-Bio. http://repobib.ubiobio.cl/jspui/bitstream/123456789/1183/1/Hizmeni_Fernandez_Julio.pd $\underline{\mathrm{f}}$

Maestre G. G. (2017). Framework de Gestión de Tecnologías de información para ciudades inteligentes: Caso Colombiano (Tesis de doctorado). Universidad del Norte. http://manglar.uninorte.edu.co/bitstream/handle/10584/8356/134019.pdf?sequence=1\&is Allowed=y

Maldonado, C. E. (Mayo de 2009). La complejidad es un problema, no una cosmovisión. UCM Revista de Investigación (13), 42-54. http://www.carlosmaldonado.org/articulos/ucm_mANIZALES.pdf

Marías, J. (1980). Historia de la filosofía (32 Ed). Biblioteca de la Revista de Occidente.

Moreno, J. (2003). Fuentes, autores y corrientes que trabajan la Complejidad. Primera Parte: Un Marco para la Complejidad. En M. A. Velilla (Comp.), Manual de Iniciación Pedagógica 
La complejidadr una perspectiva fllosófica
La complejidad: una perspectiva filosófica| Revista Virtual Universidad Católica del Norte, 64, 281-308

ISSN: 0124-5821 (En línea)

al Pensamiento Complejo (pp. 17-38). UPAEP. http://online.upaep.mx/campusvirtual/ebooks/ManualIniciacion.pdf

Morgan, H. K., y Serrato, C, S., (2016). Filosofía I. Dirección General de Bachillerato. http://www.iterra.edu.mx/centro/wp-content/uploads/2017/08/filosof\%C3\%ADa-Itodo.pdf

Morin, E. (s.f.). El paradigma de Complejidad (Tercera parte). http://www.trabajosocial.unlp.edu.ar/uploads/docs/morin_introduccion_al_pensamient o_complejo.pdf

Morin, E. (1999a). Introducción al Pensamiento Complejo (Primera Parte). Gedisa.

Morin, E. (1999b). Introducción al Pensamiento Complejo (Segunda Parte). Gedisa.

Morin, E. (2008). Complejidad restringida, complejidad general. https://upcommons.upc.edu/bitstream/handle/2099/3883/Complidad\%20restrinjida\%20co mplejidad\%20general.pdf

Osorio, F., Arnold, M., González 1., S., y Aguado, E. (2008). La nueva Teoría Social en Hispanoamérica. Introducción a la Teoría de Sistemas Constructivista. Universidad Autónoma del Estado de México. http://repositorio.uchile.cl/handle/2250/122256

Prigogine, I., y Stengers, I. (1997). La nueva alianza, metamorfosis de la ciencia (M. G. Velarde, Trad.). Alianza Editorial.

Rauch, J. E. (1993). Productivity Gains from Geographic Concentration of Human Capital: Evidence from the Cities. Journal of Urban Economics, 34(3), 380400. https://doi.org/10.1006/juec.1993.1042

Rodríguez Zoya, L. G., y Aguirre, J. L. (2011). Teorías de la Complejidad y Ciencias Sociales: Nuevas Estrategias Epistemológicas y Metodológicas. Nómadas. Revista Crítica de $\begin{array}{llll}\text { Ciencias } & \text { Sociales } & \text { 30(2). }\end{array}$ https://revistas.ucm.es/index.php/NOMA/article/view/36562/35396

Shannon, C. (July-October, 1948). A mathematical theory of communication. The Bell SystemTechnical Journal, 27: $379-423$. http://people.math.harvard.edu/ ctm/home/text/others/shannon/entropy/entropy.pdf

Shannon, C. E., \& Weaver, W. (1949). The Mathematical Theory of Communication. University of Illinois Press. 
La complejidad una perspectiva fflosófica
La complejidad: una perspectiva filosófica| Revista Virtual Universidad Católica del Norte, 64, 281-308

ISSN: 0124-5821 (En línea)

Sotolongo Codina, P., y Delgado Díaz, C. (2006). La complejidad y el diálogo transdisciplinario de saberes. En C. Latinoamericano (Ed.), La revolución contemporánea del saber y la complejidad social. Hacia unas ciencias sociales de nuevo tipo (pp. 65-77). http://bibliotecavirtual.clacso.org.ar/ar/libros/campus/soto/Capitulo\%20IV.pdf

Soublette, G. (1990). Tao Te King, Libro del Tao y de su Virtud Lao Tse. Editorial cuatro Vientos. https://www.academia.edu/30057505/TAO_TE_KING_Gast\%C3\%B3n_Soublette

Souza Santos, B. (2010). Desescolarizar el saber, reinventar el poder. Ediciones Trilce. https://periferiaactiva.files.wordpress.com/2018/03/descolonizar-el-saber_final-de-souzasantos.pdf

Turing, A. M. (1950). Computing Machinery and Intelligence (Vol. 59). Oxford University Press on behalf of the Mind Association. http://phil415.pbworks.com/f/TuringComputing.pdf

Wiener, N. (1948). Cybernetics, or Control and Communication in the Animal and the Machine. MIT Press.

Wiener, N. (1958). Cibernética y Sociedad (J. N. Cerro, Trad.) Sudamérica. https://catedraepistemologia.files.wordpress.com/2015/09/248631084-norbert-wienercibernetica-y-sociedad1.pdf

Werner, J. (1952). La teología de los primeros filósofos griegos. Fondo de Cultura Económica. https://bibliotecasaomiguel.files.wordpress.com/2013/03/jaeger-la-teologia-de-losprimeros-filosofos-griegos-ocr.pdf

Wilhelm, R. (s.f.). I CHING El Libro de los Cambios (Vol. Libro 1). http://puntocritico.com/ausajpuntocritico/documentos/__Ching.pdf

Wilhelm, R. (2007). I Ching. El Libro de las Mutaciones (32 Ed.). Edhasa.

Wilhelm, R. (2018.). I CHING. El Libro de los Cambios. Círculo de lectores. https://es.scribd.com/document/394143087/Richard-Wilhelm-I-Ching-Version-Completa

Zárate, C. L. (2010). La complejidad como referente teórico en la sistematización de experiencias educativas. Revista Virtual Universidad Católica del Norte, (29), 33-48. https://revistavirtual.ucn.edu.co/index.php/RevistaUCN/article/view/69/144 